\section{Interfacial Passivation Engineering of Perovskite Solar Cells with Fill Factor over $82 \%$ and Outstanding Operational Stability on n-i-p Architecture}

Bowen Yang, ${ }^{*} \bigcirc$ Jiajia Suo, ${ }^{\bigcirc}$ Francesco Di Giacomo,* Selina Olthof, Dmitry Bogachuk, YeonJu Kim, Xiaoxiao Sun, Lukas Wagner, Fan Fu, Shaik M. Zakeeruddin, Andreas Hinsch, Michael Grätzel, Aldo Di Carlo, and Anders Hagfeldt*

Cite This: ACS Energy Lett. 2021, 6, 3916-3923

Read Online

ACCESS 1

Llll Metrics \& More

回 Article Recommendations

Supporting Information

ABSTRACT: Tremendous efforts have been dedicated toward minimizing the open-circuit voltage deficits on perovskite solar cells (PSCs), and the fill factors are still relatively low. This hinders their further application in large scalable modules. Herein, we employ a newly designed ammonium salt, cyclohexylethylammonium iodide (CEAI), for interfacial engineering between the perovskite and hole-transporting layer (HTL), which enhanced the fill factor to $82.6 \%$ and consequent PCE of $23.57 \%$ on the target device. This can be associated with a reduction of the trap-assisted recombination rate at the 3D perovskite surface, via formation of a $2 \mathrm{D}$ perovskite interlayer. Remarkably, the property of the $2 \mathrm{D}$ perovskite interlayer along with the cyclohexylethyl group introduced by CEAI treatment also determines a pronounced enhancement in the surface hydrophobicity, leading to an outstanding stability of over $96 \%$ remaining efficiency of the passivated
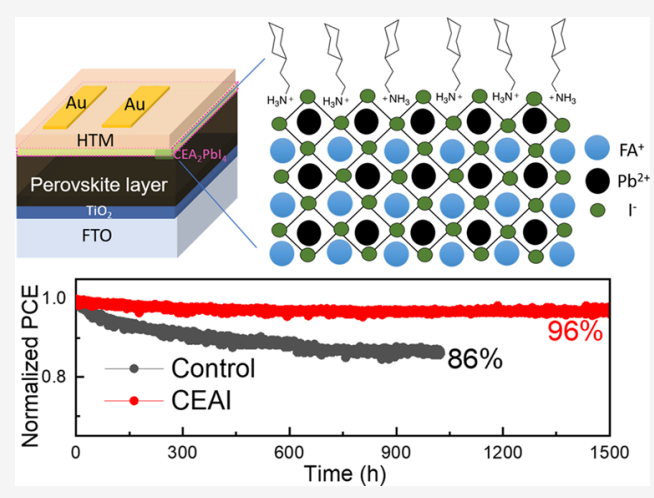
devices under maximum power point tracking with one sun illumination under $\mathrm{N}_{2}$ atmosphere at room temperature after $1500 \mathrm{~h}$.

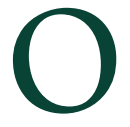

rganic-inorganic hybrid perovskite solar cells (PSCs) are one of the emerging photovoltaic (PV) technologies. Within only the past several years they have achieved photocurrent efficiencies (PCEs) similar to or even higher than those of established PV technologies, from $3.8 \%$ in 2009 up to a certified PCE of $25.5 \%$ in 2021 in a n-i-p configuration, ${ }^{1-4}$ making it an excellent and promising candidate for the future PV market. However, their inherent properties and the commonly used solution-processing technique result in a significant number of defects at grain boundaries and interfaces to adjacent functional layers. The resulting trap states can lead to undesirable hysteresis, inferior performance, and instability which hinder these remarkable photoabsorber materials from being further scaled up and commercialized in the PV market. ${ }^{5-8}$ Intensive research efforts have been devoted to address these issues, for example, by developing passivation agents to improve the photovoltaic performance and long-term stability. ${ }^{9,10}$ These include organic molecules, ${ }^{11-13}$ polymers, ${ }^{14,15}$ ionic liquids, ${ }^{16,17}$ and ammonium salts. ${ }^{18-21}$ Among these, two-dimensional (2D) perov- skites containing larger cations have attracted substantial interest owing to their outstanding stability, especially under moisture conditions, which is important in scaling-up and future commercialization. ${ }^{22}$ Recently, forming thin Ruddlesden-Popper 2D perovskite film on top of the 3D lightabsorbing perovskite layer has been developed as an excellent strategy to fabricate highly efficient PSCs. ${ }^{23-29}$ The formation of a $2 \mathrm{D}$ layer can effectively passivate the perovskite defects at the interface between the perovskite and hole transporting layers, leading to an improvement in device performance and meanwhile presenting outstanding long-term stability due to their hydrophobicity features. For example, Liu et al. employed F-rich pentafluorophenylethylammonium iodide (FEAI) on

Received: August 26, 2021

Accepted: October 11, 2021

Published: October 15, 2021 
the surface of $3 \mathrm{D}$ perovskite, achieving perovskite solar cells with over $22 \%$ efficiency and outstanding stability in humid conditions. $^{23} \mathrm{Kim}$ et al. compared different lengths of alkylammonium iodide, achieving a certified efficiency of $22.9 \%$ of the $2 \mathrm{D} / 3 \mathrm{D}$ perovskite solar cells with octylammonium iodide (OAI) treatment. ${ }^{24}$ However, most efforts have been dedicated to minimizing the open-circuit voltage deficits and fill factors remain relatively low, especially compared to $\mathrm{p}$ i-n configurations; this limitation stands in the way for their further application in large scalable modules. ${ }^{30}$

Herein, we develop and implement a novel ammonium salt, cyclohexylethylammonium iodide (CEAI), which contains a "chair" conformation cyclohexane and an equatorial substituted ethylammonium group, ${ }^{31}$ as shown in Figure 1.<smiles>NCCC1CCCCC1</smiles>

CEAI<smiles>NCCc1ccccc1</smiles>

PEAI
Figure 1. Chemical structures of CEAI and PEAI.

Compared with other well-established passivation salts containing benzene rings, such as PEAI (structure shown in Figure 1), weaker intramolecular interaction of cyclohexanes from CEAI will prevent undesirable aggregation, ${ }^{19}$ leading to a more uniform film and reduced nonradiative recombination, via the formation of a $2 \mathrm{D}$ perovskite interlayer between the $3 \mathrm{D}$ perovskite and the HTL. As a result, we achieved a target champion device with a PCE of $23.57 \%$ (stabilized at $23.4 \%$ ), $J_{\mathrm{SC}}$ of $25.04 \mathrm{~mA} / \mathrm{cm}^{2}, V_{\mathrm{OC}}$ of $1.137 \mathrm{~V}$, and an outstanding FF of $82.6 \%$. Remarkably, the obtained 2D/3D PSCs with CEAI treatment also exhibit excellent long-term stability under operational condition with one sun illumination in $\mathrm{N}_{2}$ atmosphere, which retains an impressive $96 \%$ of its initial PCE value after aging for $1500 \mathrm{~h}$. In addition, the optimized devices also exhibit superior stability while being exposed to high relative humidity $(\mathrm{RH})$ of $40 \pm 20 \%$ under dark conditions, retaining $98 \%$ of its initial PCE after $600 \mathrm{~h}$ of aging.

On the basis of our previous work, ${ }^{20,21}$ the mixed-cation and mixed-halide perovskite composition $\mathrm{Cs}_{0.05} \mathrm{MA}_{0.1} \mathrm{FA}_{0.85} \mathrm{PbI}_{2.9} \mathrm{Br}_{0.1} \cdot 0.05 \mathrm{PbI}_{2}$ was employed resulting in high quality perovskite films with superior phase stability. The perovskite films were formed by a one-step antisolvent crystallization method and annealed at $100{ }^{\circ} \mathrm{C}$ for $1 \mathrm{~h}$. Posttreatment was performed by dropping the solution of CEAI in isopropyl alcohol (IPA) on top of the perovskite film and sequentially annealing it at $100{ }^{\circ} \mathrm{C}$ for $10 \mathrm{~min}$.

The surface morphologies of the perovskite films with and without CEAI treatment were observed by high-resolution scanning electron microscopy (HR-SEM) and atomic force microscopy (AFM), as shown in Figure 2. The pristine perovskites in Figure 2a,c display a compact polycrystalline film with some crystals having higher secondary electron current densities (which appear brighter), suggesting the presence of excessive $\mathrm{PbI}_{2}{ }^{32}$ which is prone to reside mainly on the grain boundaries and the film surface. However, the additional $\mathrm{PbI}_{2}$ disappears after the post-treatment with CEAI and a surface layer formed with a significantly reduced surface roughness, as shown in Figure 2b,d. In comparison with other most commonly used ammonium salts, such as PEAI (Figure S1), the perovskite film treated with CEAI even presents better homogeneity owning to the weaker intermolecular interaction of CEAI. To analyze the composition of the newly formed layer, X-ray diffraction (XRD) measurements were carried out as presented in Figure 2e. In comparison with the pristine perovskite film, new diffraction peaks of the CEAI treated perovskite film appear at $4.7^{\circ}$ and $9.5^{\circ}$, which are consistent with the diffraction pattern of the $2 \mathrm{D}$ perovskite $\mathrm{CEA}_{2} \mathrm{PbI}_{4}$. Meanwhile, the $\mathrm{PbI}_{2}$ peak, shown at $12.6^{\circ}$ in the control film, disappears after the post-treatment of CEAI. In addition, by comparison of the XRD pattern with the film of CEAI, it is
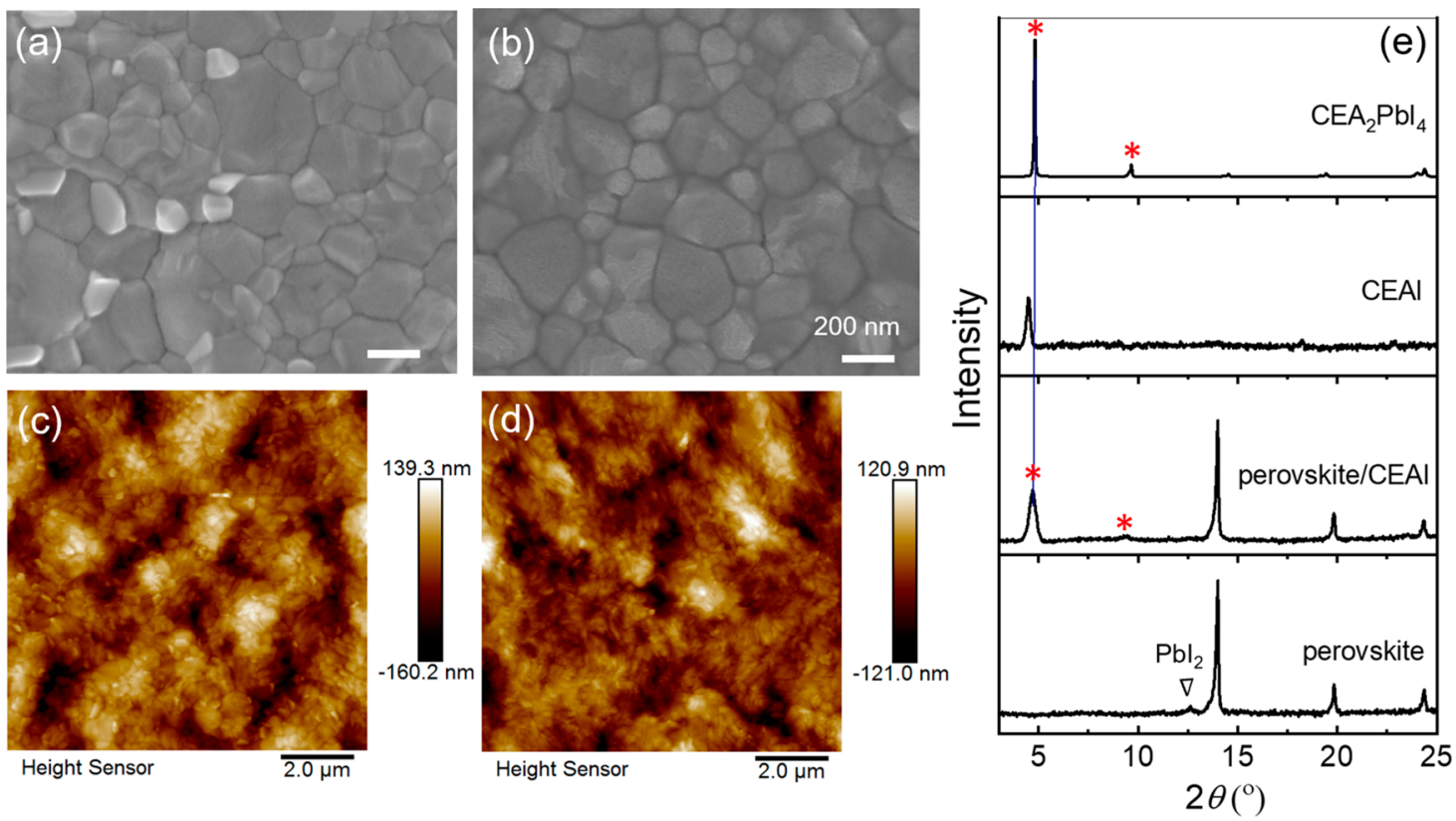

Figure 2. Surface morphology and crystal phases. Top-view SEM images of (a) pristine perovskite and (b) CEAI-treatment film. AFM images of (c) pristine perovskite and (d) CEAI-treated perovskite film. (e) XRD patterns of the pristine perovskite film, CEAI-treated perovskite film, CEAI film, and $\mathrm{CEA}_{2} \mathrm{PbI}_{4}$ film. 

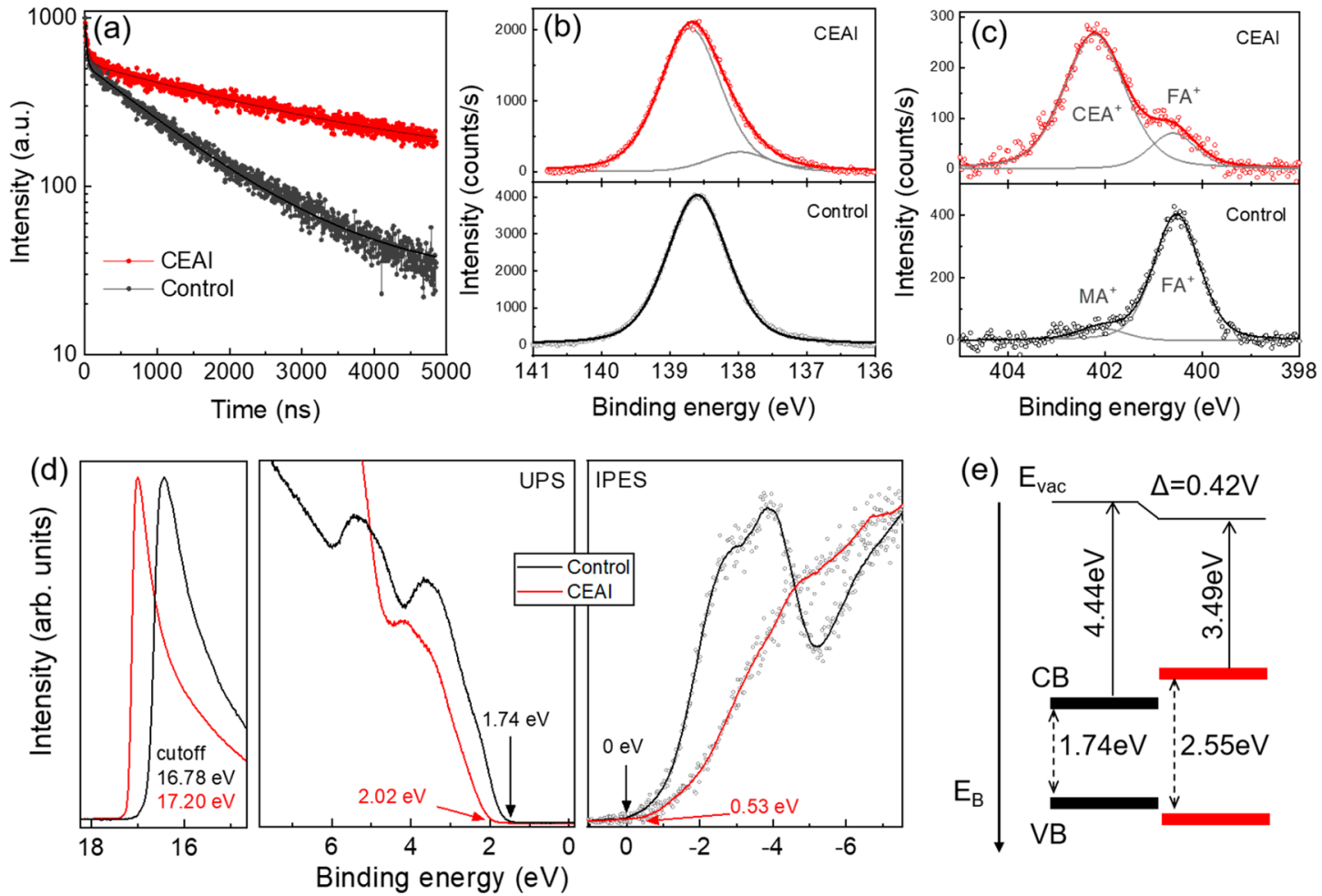

Figure 3. (a) TRPL of the perovskite film with and without CEAI treatment. Photoelectron spectroscopy data: XPS core level signals of (b) the $\mathrm{Pb} 4 \mathrm{f}_{7 / 2}$ and (c) N $1 \mathrm{~s}$ of the perovskite film with (red) and without (black) CEAI treatment; in (c) the cation species are indicated from which the core level signals originate. (d) Combined UPS and IPES spectra of the perovskite film with and without CEAI treatment. The arrows indicate the onsets of the VB and CB. (e) Schematic energy level alignment between the perovskite and the 2D capping layer, extracted from the values determined in $(\mathrm{d})$.

evidenced that instead of presenting a layer with only CEAI, an additional $2 \mathrm{D}$ perovskite layer was formed through a reaction between the remaining $\mathrm{PbI}_{2}$ from the pristine perovskite and the newly introduced CEAI. Furthermore, as shown in the grazing-incidence wide-angle X-ray scattering (GIWAXS) measurement in Figure S2, additional Debye-Scherrer rings at low angles for $2 \mathrm{D}$ perovskite reveal a preferential crystal orientation with out-of-plane direction.

Steady-state photoluminescence (PL) and time-resolved photoluminescence (TRPL) decay measurements were carried out to examine the charge recombination behavior of the perovskite film with and without CEAI treatment, as illustrated in Figure S3 and Figure 3a, respectively. A pronounced enhancement in PL intensity is observed upon CEAI treatment. Remarkably, a new feature occurs at around 500 $\mathrm{nm}$ wavelength in the PL spectra for the CEAI-treated perovskite film, which is attributed to the crystallization of the very thin $2 \mathrm{D}$ layered perovskite on top. This is in agreement with the results obtained by XRD and GIWAXS measurements as discussed above. In addition, the CEAItreated perovskite film presents a slower photoluminescence decay compared with the pristine perovskite film, as shown in Figure 3a, indicating reduced trap states and significantly suppressed nonradiative recombination upon CEAI treatment. $^{19,33}$

X-ray photoelectron spectroscopy (XPS), ultraviolet photoelectron spectroscopy (UPS), and inverse photoelectron spectroscopy (IPES) measurements were carried out to investigate the surface elemental composition, oxidation states, and energy levels of the pristine perovskite film and CEAItreated perovskite film, as presented in Figure 3. The $\mathrm{Pb} \mathrm{4f_{7/2 }}$ core level signals of the perovskite films with and without CEAI treatment are shown in Figure $3 \mathrm{~b}$. The main peak is located at approximately $138.6 \mathrm{eV}$ in both cases. However, an additional peak at $138.0 \mathrm{eV}$ emerges after the CEAI treatment, which can be associated with the binding $\mathrm{Pb}$ in the $2 \mathrm{D}$ perovskite surface layer. ${ }^{34}$ The two different organic cation species can be clearly distinguished in the $\mathrm{N}$ 1s signal shown in Figure $3 c$, due to the difference in binding energy. In the pristine perovskite film, the spectrum is dominated by a $\mathrm{FA}^{+}$ related peak at $400.5 \mathrm{eV}$, while the low amount of $\mathrm{MA}^{+}$leads to a weak signal located at $402.1 \mathrm{eV}$. Both of these values are in good agreement with literature. ${ }^{35}$ Upon treatment with CEAI, a new feature at $402.2 \mathrm{eV}$ dominates the spectrum, which now originates from nitrogen in the $\mathrm{CEA}^{+}$cation. The $\mathrm{FA}^{+}$feature can still be detected; however, it is reduced in intensity by a factor of approximately 6. This suggests that a new $\mathrm{FA}^{+}$deficient layer is formed on the top of the pristine perovskite film due to the introduction of CEAI. By use of UPS and IPES, the relative positions of the valence and conduction bands (VB, CB) of the perovskite film with and without CEAI treatment can be extracted using a linear extrapolation of the density of states at the onsets, as shown in Figure 3d. We find a reduction in work function by $420 \mathrm{meV}$ for the CEAI treated perovskite film, as well as a widening of the bandgap due to the 2D layer formed on top; this likely helps to suppress the charge recombination at the interface between the perovskite and HTL. The resulting schematic energy level diagram is presented in Figure 3e.

To evaluate the influence of CEAI on the photovoltaic performances, PSCs with the n-i-p architecture of FTO/cp$\mathrm{TiO}_{2} / \mathrm{mp}-\mathrm{TiO}_{2} / 3 \mathrm{D}$ perovskite/passivation layer/spiro-MeO- 

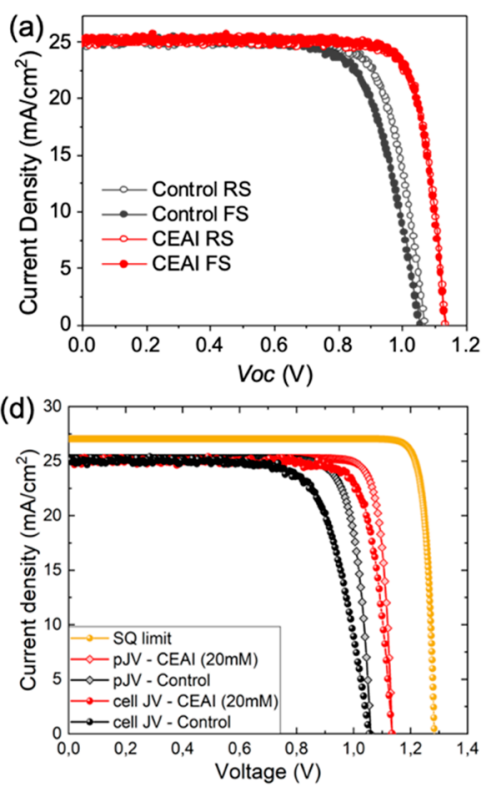
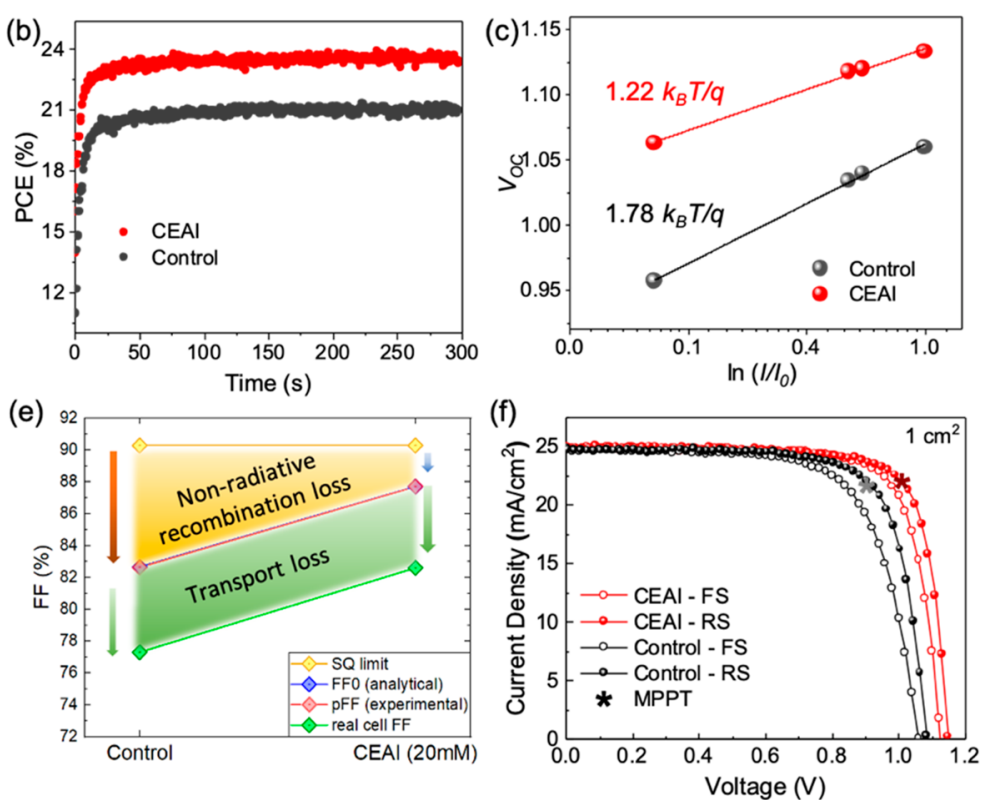

Figure 4. (a) $J-V$ curves of the control (black) and CEAI-treated (red) devices, where reverse scan (RS) and forward scan (FS) are indicated as open symbols and solid symbols, respectively. (b) Power output of the device employing CEAI treatment and the control devices at maximum power point as a function of time. (c) $V_{\mathrm{OC}}$ versus logarithm of light intensity of the PSCs with and without CEAI treatment devices. (d) $J-V$ curves of an ideal device with a bandgap of $1.56 \mathrm{eV}$ limited only by the radiative recombination (Shockley-Quisser limit), pseudo- $J V$ curves (constructed from the light-intensity-dependent $V_{\mathrm{OC}}$ measurement) of cells with and without CEAI passivation, and their actual $J-V$ curves (the area in between represents transport loss). (e) FF in the case of radiative limit for devices with this bandgap, theoretical FF $\left(\mathrm{FF}_{0}\right)$ calculated from eq 1 and experimentally obtained pseudo-FF, both of which demonstrate the FF in the absence of charge transport losses, as well as the FF of the actual devices. (f) $J-V$ curves of the champion $1 \mathrm{~cm}^{2}$ devices measured with the four-wire split method. The star indicates the $J_{\text {MPP }}$ and the $V_{\text {MPP }}$ after $60 \mathrm{~s}$ of MPPT.

$\mathrm{TAD} / \mathrm{Au}$ were fabricated with different CEAI concentrations (10 $\mathrm{mM}, 20 \mathrm{mM}$, and $30 \mathrm{mM}$ ), as shown in Figure S4. Compared to the control devices without passivation treatment, improvements in all photovoltaic parameters are observed by CEAI treatment. Among those, the devices employing $20 \mathrm{mM}$ CEAI treatment resulted in the best performance, particularly regarding the $V_{\mathrm{OC}}$ and $\mathrm{FF}$, leading to a significant increase in overall PCE. $J-V$ curves of the champion devices with and without CEAI treatment are shown in Figure 4a, and the corresponding PV parameters are listed in Table 1 . A champion cell with a PCE of $23.57 \%$ is achieved,

Table 1. Champion Photovoltaic Parameters of PSCs with and without CEAI Treatment

\begin{tabular}{|c|c|c|c|c|c|}
\hline & $J_{\mathrm{sc}}\left(\mathrm{mA} / \mathrm{cm}^{2}\right)$ & $V_{\mathrm{OC}}(\mathrm{V})$ & FF (\%) & PCE (\%) & $\mathrm{HI}^{c}(\%)$ \\
\hline control-RS ${ }^{a}$ & 25.12 & 1.074 & 77.8 & 20.99 & 6.5 \\
\hline control-FS ${ }^{b}$ & 25.09 & 1.065 & 73.4 & 19.61 & \\
\hline CEAI-RS & 25.04 & 1.137 & 82.6 & 23.57 & 1.5 \\
\hline CEAI-FS & 24.97 & 1.133 & 82.1 & 23.21 & \\
\hline
\end{tabular}

with a $J_{\mathrm{SC}}$ of $25.04 \mathrm{~mA} / \mathrm{cm}^{2}, V_{\mathrm{OC}}$ of $1.137 \mathrm{~V}$, and a remarkable FF of $82.6 \%$ in the reverse scan direction. The stabilized power output was carried out by maximum power point tracking (MPPT) for $5 \mathrm{~min}$, and the final stabilized PCE of the champion device is $23.4 \%$, shown in Figure $4 \mathrm{~b}$. The photocurrent density $\left(J_{\mathrm{SC}}\right)$ shows a comparable value of around $25 \mathrm{~mA} / \mathrm{cm}^{2}$ and the corresponding incident photon-tocurrent efficiency (IPCE) is presented in Figure S5, where the integrated $J_{\mathrm{SC}}$ is well aligned with $J_{\mathrm{SC}}$ from $J-V$ curves. In addition, negligible hysteresis is observed between the forward and reverse scans of the CEAI treated device, whereas the control device suffers from severe hysteresis. Moreover, the ideality factor $(n)$ was measured to examine the effect of CEAI treatment on the Shockley-Read-Hall recombination of the device, as shown in Figure 4c. It is observed that the slope of $V_{\text {OC }}$ versus the natural logarithm of light intensity for PSC treated with CEAI $\left(1.22 k_{\mathrm{B}} T / q\right)$ is smaller than that of the control device without passivation treatment $\left(1.78 k_{\mathrm{B}} T / q\right)$, indicating a suppressed trap-assisted charge recombination through CEAI treatment. ${ }^{36,37}$ The reduction of nonradiative recombination can also be assessed by studying the electroluminescence quantum efficiency $\left(\mathrm{EL}_{\mathrm{EQE}}\right)$ of CEAI treated devices compared to the control. As shown in Figure S6, the $\mathrm{EL}_{\mathrm{EQE}}$ increases from $0.3 \%$ to $8 \%$, in accordance with the large increase of $V_{\mathrm{OC}}{ }^{38}$ Similarly, a transient photovoltage measurement allowed study of the increase of the charge lifetime in a device, demonstrating the longer lifetime in CEAI treated devices, shown in Figure S7. It is worth noting that the devices passivated with CEAI show more improvement in device performance compared to those passivated with PEAI, as illustrated in Figure S4.

The above results indicate that CEAI treatment can effectively prolong charge carrier lifetime and reduce the nonradiative recombination rate, which results in the expected improvements not only of $V_{\mathrm{OC}}$ and diode ideality factor $(n)$ but also of FF. Therefore, to gain more insight into the origins of FF improvement by CEAI treatment, we differentiate between the FF losses caused by the nonradiative recombination and by charge transport. To do that, we calculate the theoretical FF in the absence of charge-transport losses $\left(\mathrm{FF}_{0}\right)$ coupled with light-intensity-dependent measurement of $J_{\mathrm{SC}}$ and $V_{\mathrm{OC}}$ values to construct a pseudo- $J V$ curve as shown in 

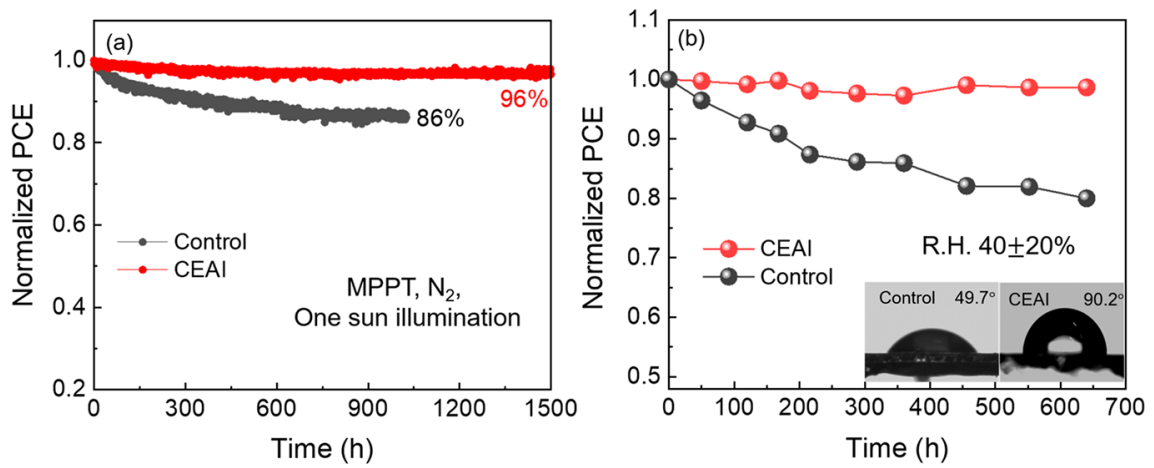

Figure 5. Long-term stability of the unencapsulated control device (black) and the optimized device with CEAI treatment (red) under (a) MPPT in $\mathrm{N}_{2}$ atmosphere with one sun illumination at $25^{\circ} \mathrm{C}$; (b) storage conditions with $\mathrm{RH}$ of $40 \pm 20 \%$ in dark at room temperature, where inset images are the contact angle of water droplet on perovskite film with and without CEAI treatment.

Figure $4 \mathrm{~d}$, which represents a $J-V$ curve without charge transport losses. More detailed discussion on the calculation of $\mathrm{FF}_{0}$ and pseudo $J-V$ curve can be found in the Supplementary Note 1. The obtained pseudo-FF ( $\mathrm{pFF}$ ) of control cells and cells with $20 \mathrm{mM}$ CEAI treatment are $82.6 \%$ and $87.7 \%$, respectively, which correlate remarkably well with the calculated $\mathrm{FF}_{0}$, which was determined to be $82.7 \%$ and $87.7 \%$ for control and CEAI-treated devices, respectively.

Comparing these values with the theoretical maximum fill factor $\mathrm{FF}_{\max }$ obtained from the Shockley-Queisser approximation (more details can be found in Supplementary Note 2), it can be seen that devices with the optimal CEAI-treatment show exceptionally low FF losses due to nonradiative recombination of $<3 \%_{\mathrm{abs}}$, while in control devices the nonradiative recombination loss is close to $8 \%$ abs (Figure $4 \mathrm{e}$ ). We note that the difference between the $\mathrm{pFF}\left(\right.$ or $\mathrm{FF}_{0}$ ) and the current-voltage FF is nearly the same for both device configurations with $5.1 \%$ abs for CEAI-treated and $5.3 \%$ abs for control devices. This clearly indicates that CEAI treatment does not cause any additional charge transport hindrance while effectively passivating the nonradiative recombination sites. We highlight that in the case of control cells, nonradiative recombination is in fact the major FF loss, which can be strongly reduced by interfacial treatment with CEAI. Hence, we attribute the increase in FF in the passivated cells entirely to the reduction of trap-assisted recombination rate at $3 \mathrm{D}$ perovskite surface, via formation of low-dimensional $\mathrm{CEA}_{2} \mathrm{PbI}_{4}$ $2 \mathrm{D}$ perovskite as mentioned above.

To further assess the quality of CEAI-treated cells, we fabricated devices with active areas of $1 \mathrm{~cm}^{2}$, with summarized PV parameters in Table S1. The CEAI treated devices have obtained a PCE of $20.4 \%$, while control devices resulted in an efficiency of $16.3 \%$ by using the standard measurement with two-wire method, which is depicted in the scheme shown in Figure S8. However, it is known that the fabrication of highly efficient large area cells requires uniform and shunt-free layers over the active area and the minimization of the resistive losses in the less conductive electrode (i.e., the transparent conductive oxide) to avoid a reduction of the FF. While the former requirement is mainly related to the fabrication protocol, the latter is strongly influenced by the design of the cell and by the method used for contacting and measuring the device. We can break down the resistive losses among the ones in the active area (intrinsic losses) and the ones in the region that connect the active area to the measurement system (extrinsic losses). Intrinsic losses are a constituent part of the device and will impact the performance of large area devices such as modules and panels. For example, increasing the cell width of a module from 3 to $10 \mathrm{~mm}$ will cause a $5 \%$ reduction of FF, and this loss cannot be avoided for large cells. ${ }^{39}$ On the other hand, extrinsic losses are particular for this configuration and are not present in optimized modules: the design of modules is aimed to reduce the ohmic losses by a series connection of narrow cells. For this reason, the extrinsic losses should not be taken in consideration when evaluating large area cells as an intermediate step toward modules. These losses can be significant since highly efficient PSC of $1 \mathrm{~cm}^{2}$ can have a large $I_{\mathrm{MPP}}$ of about $23 \mathrm{~mA}$, meaning that a $5 \Omega$ resistance will cause a drop of $115 \mathrm{mV}$ in the $V_{\text {MPP. }}$. However, it is possible, as proved in this study, to eliminate ohmic losses by using different configurations of a four-wire measurement, which acting on the measurement setup allows the evaluation of highly efficient $1 \mathrm{~cm}^{2}$ cells without the need for the optimization of the cell layout. A scheme of $1 \mathrm{~cm}^{2}$ devices and the contacting schemes for the measurements are shown in Figure S8. The minimization of the resistive losses can be obtained by placing the two negative contacts on two different sides of the active area (either on the opposite sides or on adjacent sides), as illustrated in Figure S9. As expected, the change of the position of the contact is only affecting the FF while it is not changing the $V_{\mathrm{OC}}$ or the $J_{\mathrm{SC}}$. It is possible to achive large improvement in FF by using the four-wire split measurement. With this measurement, a large area CEAItreated cell displayed a $\mathrm{PCE}_{\mathrm{MPPT}}$ of $22.4 \%$ and remarkable $\mathrm{FF}$ of $78.2 \%$. Meanwhile, as shown in Figure $4 \mathrm{f}$ and Table S1, the cell still retains a high $V_{\mathrm{OC}}$ of $1.149 \mathrm{~V}$ and a $J_{\mathrm{SC}}$ of $24.9 \mathrm{~mA} /$ $\mathrm{cm}^{2}$. Even if there is a small hysteresis in the $J V$ curve, the MPPT confirmed the results obtained with the reverse scan (see Figure S10). The CEAI treatemt showed the same effect on $1 \mathrm{~cm}^{2}$ cells, by increasing the $V_{\mathrm{OC}}$, the FF and reducing the hysteresis. Another advantage of this method is the possibility of using the $J V$ curves of $1 \mathrm{~cm}^{2}$ as input for simulating the efficiency of a module. As an example (see Figure S11 for additional parameters), a CEAI treated module could achieve an efficiency on aperture area of over $21 \%$.

Finally, long-term stability of the devices with and without CEAI treatment was monitored under maximum power point tracking in nitrogen atmosphere at room temperature under constant full sun illumination for more than $1000 \mathrm{~h}$. As shown in Figure 5a, the unencapsulated control device exhibits already $14 \%$ loss in normalized PCE after $1000 \mathrm{~h}$ aging under operational conditions, whereas the device passivated with 
CEAI remains at an impressive stability of $96 \%$ from its initial PCE value after $1500 \mathrm{~h} \mathrm{MPPT}$. In addition, the property of the newly formed $2 \mathrm{D}$ perovskite along with the cyclohexylethyl group introduced by CEAI treatment determines a pronounced enhancement in the surface hydrophobicity, compared to the bare perovskite. Accordingly, the contact angle measurements of the perovskite film with and without CEAI treatment are shown in Figure 5b (inset), where the CEAI treated film shows excellent hydrophobicity. Consequently, the shelf life stability in humid environment was investigated. The device performance was monitored while exposing them in ambient condition with relative humidity (RH) of $40 \pm 20 \%$ under dark conditions. As shown in Figure $5 \mathrm{~b}$, after over $600 \mathrm{~h}$ of aging, the PCE of the control device suffers a rapid decay of over $20 \%$ from its initial value, whereas, in sharp contrast, the target devices with CEAI treatment exhibits outstanding long-term stability under the same conditions, which still retains about 98\% PCE of its initial value.

In summary, efficient and operational stable PSCs have been demonstrated by interfacial engineering with CEAI treatment. We attribute the simultaneous enhancement of $V_{\mathrm{OC}}$ and FF to the reduction of nonradiative recombination losses at the $3 \mathrm{D}$ perovskite surface, via the formation of a $2 \mathrm{D}$ perovskite interlayer, which also determines the outstanding stability of the devices under various aging conditions. The passivated cells exhibited a long operational stability, by retaining $96 \%$ of the starting efficiency after $1500 \mathrm{~h}$ of maximum power point tracking at full sun illumination. More interestingly, by comparing with the standard two-wire method, we proposed a four-wire split method for $I V$ measurements, which has been proved to be able to largely minimize the intrinsic series resistance losses of a solar cell. Such measurement setup allows the evaluation of highly efficient $1 \mathrm{~cm}^{2}$ cells without the need for the optimization of the cell layout and can ease the evaluation of large area cells as an intermediate step for fabricating highly efficient modules.

\section{ASSOCIATED CONTENT}

\section{SI Supporting Information}

The Supporting Information is available free of charge at https://pubs.acs.org/doi/10.1021/acsenergylett.1c01811.

Synthesis of CEAI, PSCs devices fabrication, and characterization, top-view SEM image of PEAI treated perovskite film, GIWAXS images, steady-state PL, statistical box charts of device photovoltaic parameters, IPCE and integrated photocurrent density, EL spectrum, TPV decay time, champion photovoltaic parameters of 1 $\mathrm{cm}^{2}$ PSCs, schemes of two-wire and four-wire configuration, $J-V$ curevs of $1 \mathrm{~cm}^{2}$ PSCs, MPPT of 1 $\mathrm{cm}^{2}$ PSCs, and simulated maximum efficiency by fourwire split measurement (PDF)

\section{AUTHOR INFORMATION}

\section{Corresponding Authors}

Bowen Yang - Laboratory of Photomolecular Science, Institute of Chemical Sciences and Engineering, School of Basic Sciences, Ecole Polytechnique Fédérale de Lausanne, CH1015 Lausanne, Switzerland; Ångström Laboratory, Department of Chemistry, Uppsala University, SE-75120 Uppsala, Sweden; 이이.org/0000-0002-9471-3452; Email: bowen.yang@kemi.uu.se
Francesco Di Giacomo - Centre for Hybrid and Organic Solar Energy (CHOSE), Department of Electronic Engineering, University of Rome Tor Vergata, Rome 00133, Italy; (i) orcid.org/0000-0002-2489-5385; Email: francesco.di.giacomo@uniroma2.it

Anders Hagfeldt - Laboratory of Photomolecular Science, Institute of Chemical Sciences and Engineering, School of Basic Sciences, Ecole Polytechnique Fédérale de Lausanne, CH-1015 Lausanne, Switzerland; Angström Laboratory, Department of Chemistry, Uppsala University, SE-75120 Uppsala, Sweden; 이이.org/0000-0001-6725-8856; Email: anders.hagfeldt@uu.se

\section{Authors}

Jiajia Suo - Laboratory of Photomolecular Science, Institute of Chemical Sciences and Engineering, School of Basic Sciences, Ecole Polytechnique Fédérale de Lausanne, CH-1015 Lausanne, Switzerland; Ångström Laboratory, Department of Chemistry, Uppsala University, SE-75120 Uppsala, Sweden

Selina Olthof - Institute for Physical Chemistry, University of Cologne, 50939 Cologne, Germany

Dmitry Bogachuk - Fraunhofer Institute for Solar Energy Systems ISE, 79110 Freiburg, Germany; Department of Sustainable Systems Engineering (INATECH), AlbertLudwigs-Universität Freiburg, 79110 Freiburg, Germany

YeonJu Kim - Laboratory of Photomolecular Science, Institute of Chemical Sciences and Engineering, School of Basic Sciences, Ecole Polytechnique Fédérale de Lausanne, $\mathrm{CH}$ 1015 Lausanne, Switzerland

Xiaoxiao Sun - Laboratory for Thin Films and Photovoltaics, Empa-Swiss Federal Laboratories for Materials Science and Technology, 8600 Duebendorf, Switzerland

Lukas Wagner - Fraunhofer Institute for Solar Energy Systems ISE, 79110 Freiburg, Germany; Department of Sustainable Systems Engineering (INATECH), Albert-LudwigsUniversität Freiburg, 79110 Freiburg, Germany; (1) orcid.org/0000-0002-6883-5886

Fan Fu - Laboratory for Thin Films and Photovoltaics, EmpaSwiss Federal Laboratories for Materials Science and Technology, 8600 Duebendorf, Switzerland; (1) orcid.org/ 0000-0002-3647-4086

Shaik M. Zakeeruddin - Laboratory of Photonics and Interfaces, Institute of Chemical Sciences and Engineering, School of Basic Sciences, Ecole Polytechnique Fédérale de Lausanne, CH-1015 Lausanne, Switzerland

Andreas Hinsch - Fraunhofer Institute for Solar Energy Systems ISE, 79110 Freiburg, Germany; 이이이.org/00000001-7336-3599

Michael Grätzel - Laboratory of Photonics and Interfaces, Institute of Chemical Sciences and Engineering, School of Basic Sciences, Ecole Polytechnique Fédérale de Lausanne, CH-1015 Lausanne, Switzerland; (1) orcid.org/0000-00020068-0195

Aldo Di Carlo - Centre for Hybrid and Organic Solar Energy (CHOSE), Department of Electronic Engineering, University of Rome Tor Vergata, Rome 00133, Italy; Institute for Structure of the Matter, National Research Council (ISMCNR), Rome 00133, Italy; 이이.org/0000-0001-68282380

Complete contact information is available at: https://pubs.acs.org/10.1021/acsenergylett.1c01811 


\section{Author Contributions}

B.Y. and J.S. conceived the idea, prepared films, fabricated all devices, and did the characterizations. J.S. designed and synthesized the CEAI molecule. F.D.G. conceived and applied the four-wire split methods for $I V$ measurements. S.O. assisted in XPS, UPS, and IPES measurements and analysis. D.B., L.W., and A.H. performed the simulation on nonradiative recombination losses. F.D.G. performed TPV and charge lifetime measurements and analysis. X.S. assisted in AFM measurements. Y.K. performed steady state $\mathrm{PL}$ and contact angle measurements. B.Y., J.S., F.D.G., D.B., and S.O. all participated in editing the manuscript. A.H. directed the overall research. All authors read and commented on the manuscript.

\section{Author Contributions}

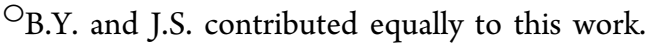

\section{Notes}

The authors declare no competing financial interest.

\section{ACKNOWLEDGMENTS}

This project has received funding from the European Union's Horizon 2020 research and innovation program under Grant Agreement 764047 and from Swiss National Science Foundation for financial support with Project 200020_185041. S.O. acknowledges funding by the German Federal Ministry for Education and Research (MUJUPO, Grant OL 462/4-1). D.B. and L.W. acknowledge the scholarship support of the German Federal Environmental Foundation (DBU). X.S. and F.F. acknowledge funding from ETH Zurich Postdoctoral Fellowship.

\section{REFERENCES}

(1) Kojima, A.; Teshima, K.; Shirai, Y.; Miyasaka, T. Organometal Halide Perovskite as Visible-Light Sensitizers for Photovoltaic Cells. J. Am. Chem. Soc. 2009, 131, 6050-6051.

(2) Kim, H.-S.; Lee, C.-R.; Im, J.-H.; Lee, K.-B.; Moehl, T.; Marchioro, A.; Moon, S.-J.; Humphry-Baker, R.; Yum, J.-H.; Moser, J. E.; Grätzel, M.; Park, N.-G. Lead Iodide Perovskite Sensitized AllSolid-State Submicron Thin Film Mesoscopic Solar Cell with Efficiency Exceeding 9\%. Sci. Rep. 2012, 2, 591.

(3) Lee, M. M.; Teuscher, J.; Miyasaka, T.; Murakami, T. N.; Snaith, H. J. Efficient Hybrid Solar Cells Based on Meso-Superstructured Organometal Halide Perovskites. Science 2012, 338, 643-647.

(4) Best Research-Cell Efficiencies Chart. https://www.nrel.gov/pv/ assets/pdfs/best-research-cell-efficiencies.20210926.pdf.

(5) Luo, D.; Su, R.; Zhang, W.; Gong, Q.; Zhu, R. Minimizing NonRadiative Recombination Losses in Perovskite Solar Cells. Nat. Rev. Mater. 2020, 5, 44-60.

(6) Tress, W. Perovskite Solar Cells on the Way to Their Radiative Efficiency Limit - Insights into a Success Story of High Open-Circuit Voltage and Low Recombination. Adv. Energy Mater. 2017, 7, 1602358.

(7) Han, T.-H.; Tan, S.; Xue, J.; Meng, L.; Lee, J.-W.; Yang, Y. Interface and Defect Engineering for Metal Halide Perovskite Optoelectronic Devices. Adv. Mater. 2019, 31, 1803515.

(8) Suo, J.; Yang, B.; Hagfeldt, A. Passivation Strategies through Surface Reconstruction toward Highly Efficient and Stable Perovskite Solar Cells on n-i-p Architecture. Energies 2021, 14, 4836.

(9) Akin, S.; Arora, N.; Zakeeruddin, S. M.; Grätzel, M.; Friend, R. H.; Dar, M. I. New Strategies for Defect Passivation in HighEfficiency Perovskite Solar Cells. Adv. Energy Mater. 2020, 10, 1903090.

(10) Aydin, E.; De Bastiani, M.; De Wolf, S. Defect and Contact Passivation for Perovskite Solar Cells. Adv. Mater. 2019, 31, 1900428.

(11) Wang, R.; Xue, J.; Wang, K.-L.; Wang, Z.-K.; Luo, Y.; Fenning, D.; Xu, G.; Nuryyeva, S.; Huang, T.; Zhao, Y.; Yang, J. Y.; Zhu, J.;
Wang, M.; Tan, S.; Yavuz, I.; Houk, K. N.; Yang, Y. Constructive Molecular Configurations for Surface-defect Passivation of Perovskite Photovoltaics. Science 2019, 366, 1509-1513.

(12) Hu, Q.; Chen, W.; Yang, W.; Li, Y.; Zhou, Y.; Larson, B. W.; Johnson, J. C.; Lu, Y.; Zhong, W.; Xu, J.; Klivansky, L.; Wang, C.; Salmeron, M.; Djurišíc, A. B.; Liu, F.; He, Z.; Zhu, R.; Russell, T. P. Improving Efficiency and Stability of Perovskite Solar Cells Enabled by A Near-Infrared-Absorbing Moisture Barrier. Joule. 2020, 4, 15751593.

(13) Cai, Y.; Cui, J.; Chen, M.; Zhang, M.; Han, Y.; Qian, F.; Zhao, H.; Yang, S.; Yang, Z.; Bian, H.; Wang, T.; Guo, K.; Cai, M.; Dai, S.; Liu, Z.; Liu, S. Multifunctional Enhancement for Highly Stable and Efficient Perovskite Solar Cells. Adv. Funct. Mater. 2021, 31, 2005776.

(14) Bi, D.; Yi, C.; Luo, J.; Décoppet, J.-D.; Zhang, F.; Zakeeruddin, S. M.; Li, X.; Hagfeldt, A.; Grätzel, M. Polymer-templated Nucleation and Crystal Growth of Perovskite Films for Solar Cells with Efficiency Greater than 21\%. Nat. Energy. 2016, 1, 16142.

(15) Kim, M.; Motti, S. G.; Sorrentino, R.; Petrozza, A. Enhanced Solar Cell Stability by Hygroscopic Polymer Passivation of Metal Halide Perovskite Thin Film. Energy Environ. Sci. 2018, 11, 26092619.

(16) Bai, S.; Da, P.; Li, C.; Wang, Z.; Yuan, Z.; Fu, F.; Kawecki, M.; Liu, X.; Sakai, N.; Wang, J. T.-W.; Huettner, S.; Buecheler, S.; Fahlman, M.; Gao, F.; Snaith, H. J. Planar Perovskite Solar Cells with Long-term Stability Using Ionic Liquid Additives. Nature 2019, 571, $245-250$.

(17) Wang, S.; Yang, B.; Han, J.; He, Z.; Li, T.; Cao, Q.; Yang, J.; Suo, J.; Li, X.; Liu, Z.; Liu, S.; Tang, C.; Hagfeldt, A. Polymeric Room Temperature Molten Salt as a Multifunctional Additive toward Highly Efficient and Stable Inverted Planar Perovskite Solar Cells. Energy Environ. Sci. 2020, 13, 5068-5079.

(18) Jiang, Q.; Zhao, Y.; Zhang, X.; Yang, X.; Chen, Y.; Chu, Z.; Ye, Q.; Li, X.; Yin, Z.; You, J. Surface Passivation of Perovskite Film for Efficient Solar Cells. Nat. Photonics 2019, 13, 460-466.

(19) Zhu, H.; Liu, Y.; Eickemeyer, F. T.; Pan, L.; Ren, D.; RuizPreciado, M. A.; Carlsen, B.; Yang, B.; Dong, X.; Wang, Z.; Liu, H.; Wang, S.; Zakeeruddin, S. M.; Hagfeldt, A.; Dar, M. I.; Li, X.; Grätzel, M. Tailored Amphiphilic Molecular Mitigators for Stable Perovskite Solar Cells with 23.5\% Efficiency. Adv. Mater. 2020, 32, 1907757.

(20) Yang, B.; Suo, J.; Mosconi, E.; Ricciarelli, D.; Tress, W.; De Angelis, F.; Kim, H.-S.; Hagfeldt, A. Outstanding Passivation Effect by a Mixed-Salt Interlayer with Internal Interactions in Perovskite Solar Cells. ACS Energy Lett. 2020, 5, 3159-3167.

(21) Suo, J.; Yang, B.; Mosconi, E.; Choi, H.-C.; Kim, Y.; Zakeeruddin, S. M.; De Angelis, F.; Grätzel, M.; Kim, H.-S.; Hagfeldt, A. Surface Reconstruction Engineering with Synergistic Effect of Mixed-Salt Passivation Treatment toward Efficient and Stable Perovskite Solar Cells. Adv. Funct. Mater. 2021, 31, 2102902.

(22) Gangadharan, D. T.; Ma, D. Searching for Stability at Lower Dimensions: Current Trends and Future Prospects of Layered Perovskite Solar Cells. Energy Environ. Sci. 2019, 12, 2860-2889.

(23) Liu, Y.; Akin, S.; Pan, L.; Uchida, R.; Arora, N.; Milić, J.; Hinderhofer, A.; Schreiber, F.; Uhl, A. R.; Zakeeruddin, S. M.; Hagfeldt, A.; Dar, M. I.; Grätzel, M. Ultrahydrophobic 3D/2D Fluoroarene Bilayer-based Water-resistant Perovskite Solar Cells with Efficiencies Exceeding 22\%. Sci. Adv. 2019, 5, No. eaaw2543.

(24) Kim, H.; Lee, S.-U.; Lee, D. Y.; Paik, M. J.; Na, H.; Lee, J.; Seok, S. I. Optimal Interfacial Engineering with Different Length of Alkylammonium Halide for Efficient and Stable Perovskite Solar Cells. Adv. Energy Mater. 2019, 9, 1902740.

(25) Yoo, J. J.; Wieghold, S.; Sponseller, M. C.; Chua, M. R.; Bertram, S. N.; Hartono, N. T. P.; Tresback, J. S.; Hansen, E. C.; Correa-Baena, J.-P.; Bulović, V.; Buonassisi, T.; Shin, S. S.; Bawendi, M. D. An Interface Stabilized Perovskite Solar Cell with High Stabilized Efficiency and Low Voltage Loss. Energy Environ. Sci. 2019, 12, 2192-2199.

(26) Jang, Y.-W.; Lee, S.; Yeom, K. M.; Jeong, K.; Choi, K.; Choi, M.; Noh, J. H. Intact 2D/3D Halide Junction Perovskite Solar Cells via Solid-phase in-plane Growth. Nat. Energy. 2021, 6, 63-71. 
(27) Ma, C.; Park, N.-G. Paradoxical Approach with a Hydrophilic Passivation Layer for Moisture-Stable, 23\% Efficient Perovskite Solar Cells. ACS Energy Lett. 2020, 5, 3268-3275.

(28) Chen, P.; Bai, Y.; Wang, S.; Lyu, M.; Yun, J.-H.; Wang, L. In Situ Growth of 2D Perovskite Capping Layer for Stable and Efficient Perovskite Solar Cells. Adv. Funct. Mater. 2018, 28, 1706923.

(29) Cho, Y.; Soufiani, A. M.; Yun, J. S.; Kim, J.; Lee, D. S.; Seidel, J.; Deng, X.; Green, M. A.; Huang, S.; Ho-Baillie, A. W. Y. Mixed 3D2D Passivation Treatment for Mixed-Cation Lead Mixed-Halide Perovskite Solar Cells for Higher Efficiency and Better Stability. Adv. Energy Mater. 2018, 8, 1703392.

(30) Peng, J.; Walter, D.; Ren, Y.; Tebyetekerwa, M.; Wu, Y.; Duong, T.; Lin, Q.; Li, J.; Lu, T.; Mahmud, M. A.; Lem, O. L. C.; Zhao, S.; Liu, W.; Liu, Y.; Shen, H.; Li, L.; Kremer, F.; Nguyen, H. T.; Choi, D.; Weber, K. J.; Catchpole, K. R.; White, T. P. Nanoscale Localized Contacts for High Fill Factors in Polymer-Passivated Perovskite Solar Cells. Science 2021, 371, 390-395.

(31) Squillacote, M.; Sheridan, R. S.; Chapman, O. L.; Anet, F. A. L. Spectroscopic Detection of the Twist-Boat Conformation of Cyclohexane. Direct Measurement of the Free Energy Difference between the Chair and the Twist-boat. J. Am. Chem. Soc. 1975, 97, 3244-3246.

(32) Chen, Q.; Zhou, H.; Song, T.-B.; Luo, S.; Hong, Z.; Duan, H.S.; Dou, L.; Liu, Y.; Yang, Y. Controllable Self-Induced Passivation of Hybrid Lead Iodide Perovskites toward High Performance Solar Cells. Nano Lett. 2014, 14, 4158-4163.

(33) Jeong, J.; Kim, M.; Seo, J.; Lu, H.; Ahlawat, P.; Mishra, A.; Yang, Y.; Hope, M. A.; Eickemeyer, F. T.; Kim, M.; Yoon, Y. J.; Choi, I. W.; Darwich, B. P.; Choi, S. J.; Jo, Y.; Lee, J. H.; Walker, B.; Zakeeruddin, S. M.; Emsley, L.; Rothlisberger, U.; Hagfeldt, A.; Kim, D. S.; Grätzel, M.; Kim, J. Y. Pseudo-halide anion engineering for $\alpha$ $\mathrm{FAPbI}_{3}$ perovskite solar cells. Nature 2021, 592, 381-385.

(34) Chen, J.; Seo, J. - Y.; Park, N.-G. Simultaneous Improvement of Photovoltaic Performance and Stability by In Situ Formation of 2D Perovskite at $\left(\mathrm{FAPbI}_{3}\right)_{0.88}\left(\mathrm{CsPbBr}_{3}\right)_{0.12} / \mathrm{CuSCN}$ Interface. $A d v$. Energy Mater. 2018, 8, 1702714.

(35) Philippe, B.; Saliba, M.; Correa-Baena, J.-P.; Cappel, U. B.; Turren-Cruz, S. H.; Grätzel, M.; Hagfeldt, A.; Rensmo, H. Chemical Distribution of Multiple Cation $\left(\mathrm{Rb}^{+}, \mathrm{Cs}^{+}, \mathrm{MA}^{+}\right.$, and $\left.\mathrm{FA}^{+}\right)$Perovskite Materials by Photoelectron Spectroscopy. Chem. Mater. 2017, 29, 3589-3596.

(36) Yang, D.; Zhou, X.; Yang, R.; Yang, Z.; Yu, W.; Wang, X.; Li, C.; Liu, S.; Chang, R. P. H. Surface Optimization to Eliminate Hysteresis for Record Efficiency Planar Perovskite Solar Cells. Energy Environ. Sci. 2016, 9, 3071-3078.

(37) Tress, W.; Yavari, M.; Domanski, K.; Yadav, P.; Niesen, B.; Correa-Baena, J.-P.; Hagfeldt, A.; Grätzel, M. Interpretation and Evolution of Open-Circuit Voltage, Recombination, Ideality Factor and Subgap Defect States during Reversible Light-soaking and Irreversible Degradation of Perovskite Solar Cells. Energy Environ. Sci. 2018, 11, 151-165.

(38) Tress, W.; Marinova, N.; Inganäs, O.; Nazeeruddin, M. K.; Zakeeruddin, S. M.; Grätzel, M. Predicting the Open-Circuit Voltage of $\mathrm{CH}_{3} \mathrm{NH}_{3} \mathrm{PbI}_{3}$ Perovskite Solar Cells Using Electroluminescence and Photovoltaic Quantum Efficiency Spectra: the Role of Radiative and Non-Radiative Recombination. Adv. Energy Mater. 2015, 5, 1400812.

(39) Di Giacomo, F.; Castriotta, L. A.; Kosasih, F. U.; Di Girolamo, D.; Ducati, C.; Di Carlo, A. Upscaling Inverted Perovskite Solar Cells: Optimization of Laser Scribing for Highly Efficient Mini-Modules. Micromachines. 2020, 11, 1127. 